\title{
Self-dual solitons in a Born-Infeld baby Skyrme model
}

\author{
Rodolfo Casana $\odot^{*}$ and André C. Santos $\odot^{\dagger}$ \\ Departamento de Física, Universidade Federal do Maranhão, 65080-805 São Luís, Maranhão, Brazil
}

(Received 21 June 2021; accepted 30 July 2021; published 10 September 2021)

\begin{abstract}
We show the existence of self-dual (topological) solitons in a gauged version of the baby Skyrme model in which the Born-Infeld term governs the gauge field dynamics. The successful implementation of the Bogomol'nyi-Prasad-Sommerfield formalism provides a lower bound for the energy and the respective self-dual equations whose solutions are the solitons saturating such a limit. The energy lower bound (Bogomol'nyi bound) is proportional to the topological charge of the Skyrme field and therefore quantized. In contrast, the total magnetic flux is a nonquantized quantity. Furthermore, the model supports three types of self-dual solitons profiles: the first describes compacton solitons, the second follows a Gaussian decay law, and the third portrays a power-law decay. Finally, we perform numerical solutions of the self-dual equations and depict the soliton profiles for different values of the parameters controlling the nonlinearity of the model.
\end{abstract}

DOI: 10.1103/PhysRevD.104.065009

\section{INTRODUCTION}

The Skyrme model [1] is a well-known low-energy field theory proposal to study nonperturbative quantum chromodynamics (QCD). This nonlinear effective field theory, defined initially in $(3+1)$ dimensions, provides some insights into the physical properties of hadrons and nuclei belonging to the realm of low-energy QCD [2]. In this framework, the physical structures emerge as topological solitons-so-called Skyrmions.

Over the years there has been remarkable progress in our understanding of Skyrme-like models, especially about its corresponding planar version, also known as the baby Skyrme model [3]. The $(2+1)$-dimensional version serves as a laboratory to study many aspects of the original Skyrme model.

Besides, the Skyrmions have also attracted the community's attention because they are used or come up in the description of various physical systems. Among them we can mention the topological quantum Hall effect [4], chiral nematic liquid crystals [5], superconductors [6], brane cosmology [7], and magnetic materials [8], including recent investigations with the Dzyaloshinskii-Moriya interaction $[9,10]$.

The Skyrme model consists of a $O(3)$ nonlinear sigmamodel term (a quadratic kinetic term), the Skyrme term

\footnotetext{
rodolfo.casana@ufma.br,rodolfo.casana@gmail.com †andre.cs@discente.ufma.br, andre_cavs@hotmail.com
}

Published by the American Physical Society under the terms of the Creative Commons Attribution 4.0 International license. Further distribution of this work must maintain attribution to the author(s) and the published article's title, journal citation, and DOI. Funded by SCOAP ${ }^{3}$. (a quartic kinetic term), and a potential (a nonderivative one). For the baby Skyrme model, the presence of such a potential is obligatory to stabilize the soliton solutions $[11,12]$, being optional in the $3+1$-dimensional version. Although the standard baby Skyrme model describes stable solitons, it does not possess a self-dual or Bogomol'nyiPrasad-Sommerfield (BPS) structure. Nevertheless, lacking the sigma model term, the so-called restricted baby Skyrme model [13] does admit BPS configurations [14].

To investigate the electric and magnetic properties of the Skyrme model, we must couple it with a $U(1)$ gauge field [15]. Soliton solutions in the gauged baby Skyrme model were first found in Ref. [16], and were later considered in Ref. [17] and by including a Chern-Simons term in Refs. [18,19]. On the other hand, in Refs. [20-22] BPS configurations (carrying magnetic flux alone) were studied in the restricted gauge baby Skyrme model. Moreover, Refs. [23-25] analyzed the self-dual solutions carrying both magnetic flux and electric charge. It is essential to point out that the restricted gauge baby Skyrme model also allows a supersymmetric extension [26-30].

In the literature, the emergence of topological defects is also a current issue in generalized or effective field theories. In particular, the new solitons may present quite different features than those found in the usual models. There are many results in models characterized by possessing nonstandard kinetic terms (e.g., Refs. [31-39]), including supersymmetric extensions [40,41]. Moreover, there have been additional applications, such as in the inflationary phase of the Universe [42], strong gravitational waves [43], tachyon matter [44], dark matter [45], black holes [46,47], and other topics $[48,49]$. 
Among several generalized or effective models, we may highlight the Born-Infeld (BI) electrodynamics [50], also dubbed Dirac-Born-Infeld theory [51]. In this elegant model, the gauge field's kinetic term is a highly nonlinear function of the Maxwell term (instead of the usual Maxwell term) that removes the divergence of the electron selfenergy appearing in classical electrodynamics. The BornInfeld-Higgs $(\mathrm{BIH})$ model can be considered one of the pioneering studies of vortex solutions within the scope of such generalized theories [52]. Later, in Ref. [53] the existence of electrically charged BIH vortices was studied through the inclusion of the Chern-Simons term. Further, the BI theory has been explored in different contexts, including topological defects [34-37,54,55], unusual properties under wave propagation [56], in gravitation and cosmology [57,58], quantum gravity [59], and supersymmetric extensions [30]. Currently, BI electrodynamics is worthy of special attention since it appears in the lowenergy limit of string/D-brane physics [33,60-63].

Thus, motivated by the versatility of the Born-Infeld term, the present manuscript aims to show the existence of the topological BPS solitons in a Born-Infeld baby Skyrme model and explore their main physical properties. We have organized the paper as follows. In Sec. II, we introduce the BI restricted baby Skyrme model and implement the BPS formalism to get the topological energy lower bound and self-dual equations that provide the soliton solutions saturating such a limit. In Sec. III, we focus our attention on rotationally symmetric configurations. We study the behavior of the profiles around boundaries, obtain the numerical solution of the BPS equations, and depict the main features of the BPS configurations. Finally, in Sec. IV we include our remarks and conclusions.

\section{THE BORN-INFELD RESTRICTED BABY SKYRME MODEL}

We consider a model describing the interaction between the restricted baby Skyrme field and a Born-Infeld gauge field defined by the Lagrangian

$$
L=E_{0} \int d^{2} \mathbf{x} \mathcal{L},
$$

where $E_{0}$ is a common factor of the energy scale which hereafter we set to be $E_{0}=1$, and $\mathcal{L}$ stands for the Lagrangian density given by

$$
\mathcal{L}=\beta^{2}(1-\mathcal{R})-\frac{\lambda^{2}}{4}\left(D_{\mu} \vec{\phi} \times D_{\nu} \vec{\phi}\right)^{2}-\mathcal{V}\left(\phi_{n}\right),
$$

where we have defined

$$
\mathcal{R}=\sqrt{1+\frac{F_{\mu \nu}^{2}}{2 \beta^{2} g^{2}}}, \quad \mathcal{V}\left(\phi_{n}\right)=\beta^{2}\left[1-U\left(\phi_{n}\right)\right] .
$$

The first contribution in Eq. (2) is the BI term, where $F_{\mu \nu}=\partial_{\mu} A_{\nu}-\partial_{\nu} A_{\mu}$ is the field-strength tensor of the $U(1)$ gauge field $A_{\mu}$, the electromagnetic coupling constant is given by $g$, and $\beta$ is the BI parameter. The second contribution is the Skyrme term minimally coupled to the $U(1)$ gauge field through the covariant derivative given by

$$
D_{\mu} \vec{\phi}=\partial_{\mu} \vec{\phi}+A_{\mu} \vec{n} \times \vec{\phi},
$$

where $\vec{\phi} \in \mathbb{S}^{2}$, a triplet of real scalar fields, represents the Skyrme field satisfying the constraint $\vec{\phi} \cdot \vec{\phi}=1$. The unitary vector $\vec{n}$ provides a preferred direction in the internal space that afterwards becomes the vacuum value of the Skyrme field, that is,

$$
\lim _{|\vec{x}| \rightarrow \infty} \vec{\phi}=\vec{n} .
$$

Last, the third term in Eq. (2), $\mathcal{V}\left(\phi_{n}\right)=\mathcal{V}(\vec{n} \cdot \vec{\phi})$, is the potential defined in Eq. (3) with the function $U\left(\phi_{n}\right)$ satisfying the condition $0<U\left(\phi_{n}\right)<1$.

We assume that all of the coupling constants are non-negative quantities. Moreover, the Skyrme field is dimensionless, the gauge field has mass dimension equal to 1 , both the BI parameter $\beta$ and the electromagnetic coupling constant $g$ have mass dimension 1, and the Skyrme coupling constant $\lambda$ has mass dimension -1 .

We now proceed to present the Euler-Lagrange equations resulting from the Lagrangian density (2). The equation of motion of the gauge field reads

$$
\partial_{\sigma}\left(\frac{1}{\mathcal{R}} F^{\sigma \mu}\right)=g^{2} j^{\mu}
$$

where $j^{\mu}=\vec{n} \cdot \vec{J}^{\mu}$ is the conserved current density with

$$
\vec{J}^{\mu}=\lambda^{2}\left[\vec{\phi} \cdot\left(D^{\mu} \vec{\phi} \times D^{\nu} \vec{\phi}\right)\right]\left(D_{\nu} \vec{\phi}\right) .
$$

Already for the Skyrme field we obtain

$$
D_{\mu} \vec{J}^{\mu}+\frac{\partial \mathcal{V}}{\partial \phi_{n}}(\vec{n} \times \vec{\phi})=0
$$

Our effort will be focused on the study of stationary solutions. Then, from Eq. (6) Gauss' law reads as

$$
\partial_{i}\left(\frac{1}{\mathcal{R}} \partial_{i} A_{0}\right)=g^{2} \lambda^{2} A_{0}\left(\vec{n} \cdot \partial_{i} \vec{\phi}\right)^{2} .
$$

We observe that the gauge condition $A_{0}=0$ identically satisfies Gauss' law, implying that the resulting configurations only carry on magnetic flux. 
Furthermore, from Eq. (6) the stationary Ampère's law gives

$$
\partial_{i}\left(\frac{B}{\mathcal{R}}\right)+\lambda^{2} g^{2}\left(\vec{n} \cdot \partial_{i} \vec{\phi}\right) Q=0
$$

where $B=F_{12}=\epsilon_{i j} \partial_{i} A_{j}$ is the magnetic field, $\mathcal{R}$ assumes the form

$$
\mathcal{R}=\sqrt{1+\frac{B^{2}}{\beta^{2} g^{2}}},
$$

and $Q \equiv \vec{\phi} \cdot\left(D_{1} \vec{\phi} \times D_{2} \vec{\phi}\right)$, which can still be expressed as

$$
Q=\vec{\phi} \cdot\left(\partial_{1} \vec{\phi} \times \partial_{2} \vec{\phi}\right)+\epsilon_{i j} A_{i}\left(\vec{n} \cdot \partial_{j} \vec{\phi}\right)
$$

The term $\vec{\phi} \cdot\left(\partial_{1} \vec{\phi} \times \partial_{2} \vec{\phi}\right)$ is related to the topological charge or topological degree (also called winding number) of the Skyrme field,

$$
\operatorname{deg}[\vec{\phi}]=-\frac{1}{4 \pi} \int d^{2} \mathbf{x} \vec{\phi} \cdot\left(\partial_{1} \vec{\phi} \times \partial_{2} \vec{\phi}\right)=k
$$

with $k \in \mathbb{Z} \backslash 0$.

Similarly, from Eq. (8) the stationary equation of motion of the Skyrme field becomes

$$
\frac{\partial U}{\partial \phi_{n}}(\vec{n} \times \vec{\phi})+\lambda^{2} \epsilon_{i j} D_{i}\left(Q D_{j} \vec{\phi}\right)=0 .
$$

In the next section we show how the BPS formalism is implemented, allowing us to obtain the energy lower bound and the self-dual equations to be satisfied by the soliton configurations saturating such a bound.

\section{A. The BPS framework}

The stationary energy density of the model (2) is

$$
\varepsilon=\beta^{2}(\mathcal{R}-U)+\frac{\lambda^{2}}{2} Q^{2},
$$

where we have used $Q^{2}=\frac{1}{2}\left(D_{i} \vec{\phi} \times D_{j} \vec{\phi}\right)^{2}$. The requirement that the energy density is null when $|\vec{x}| \rightarrow \infty$ establishes the boundary conditions satisfied by the fields of the model. Thus, from Eq. (11) the magnetic field must satisfy $\lim _{|\vec{x}| \rightarrow \infty} B=0$, implying that $\lim _{|\vec{x}| \rightarrow \infty} \mathcal{R}=1$; consequently,

$$
\lim _{|\vec{x}| \rightarrow \infty} U=1, \quad \lim _{|\vec{x}| \rightarrow \infty} Q=0 .
$$

The total energy is defined by integrating the energy density (15), so that we implement the BPS formalism by writing

$$
\begin{aligned}
E= & \int d^{2} \mathbf{x}\left[\frac{\left(B \pm \lambda^{2} g^{2} \mathcal{R} W\right)^{2}}{2 g^{2} \mathcal{R}}+\frac{\lambda^{2}}{2}(Q \mp Z)^{2}\right. \\
& \mp \lambda^{2} B W \pm \lambda^{2} Q Z-\beta^{2} U+\beta^{2} \mathcal{R} \\
& \left.-\frac{B^{2}}{2 g^{2} \mathcal{R}}-\frac{\lambda^{4} g^{2} \mathcal{R} W^{2}}{2}-\frac{\lambda^{2}}{2} Z^{2}\right]
\end{aligned}
$$

where we have introduced two auxiliary functions, namely, $W \equiv W\left(\phi_{n}\right)$ and $Z \equiv Z\left(\phi_{n}\right)$, which we shall determine later. By using the magnetic field definition and Eqs. (11) and (12) for $\mathcal{R}$ and $Q$, respectively, we arrive at

$$
\begin{aligned}
E= & \int d^{2} \mathbf{x}\left[\frac{\left(B \pm \lambda^{2} g^{2} \mathcal{R} W\right)^{2}}{2 g^{2} \mathcal{R}}+\frac{\lambda^{2}}{2}(Q \mp Z)^{2}\right. \\
& \pm \lambda^{2} Z \vec{\phi} \cdot\left(\partial_{1} \vec{\phi} \times \partial_{2} \vec{\phi}\right) \pm \lambda^{2} \epsilon_{i j}\left[\left(\partial_{j} A_{i}\right) W+A_{i} Z\left(\partial_{j} \phi_{n}\right)\right] \\
& \left.-\beta^{2} U+\frac{\beta^{2}}{2}\left(\frac{1}{\mathcal{R}}+\mathcal{R}\right)-\frac{\lambda^{4} g^{2} \mathcal{R} W^{2}}{2}-\frac{\lambda^{2}}{2} Z^{2}\right]
\end{aligned}
$$

At this point, to continue with the implementation of the BPS formalism, we perform two steps: (i) the term $\left(\partial_{j} A_{i}\right) W+A_{i} Z\left(\partial_{j} \phi_{n}\right)$ is transformed into a total derivative by setting

$$
Z=\frac{\partial W}{\partial \phi_{n}}
$$

and (ii) we require the function $U\left(\phi_{n}\right)$ to be defined as

$$
\beta^{2} U=\frac{\beta^{2}}{2}\left(\frac{1}{\mathcal{R}}+\mathcal{R}\right)-\frac{\lambda^{4} g^{2} \mathcal{R} W^{2}}{2}-\frac{\lambda^{2}}{2}\left(\frac{\partial W}{\partial \phi_{n}}\right)^{2} .
$$

This way, the total energy becomes

$$
\begin{aligned}
E= & \int d^{2} \mathbf{x}\left[\frac{\left(B \pm \lambda^{2} g^{2} \mathcal{R} W\right)^{2}}{2 g^{2} \mathcal{R}}+\frac{\lambda^{2}}{2}\left(Q \mp \frac{\partial W}{\partial \phi_{n}}\right)^{2}\right. \\
& \left. \pm \lambda^{2}\left(\frac{\partial W}{\partial \phi_{n}}\right) \vec{\phi} \cdot\left(\partial_{1} \vec{\phi} \times \partial_{2} \vec{\phi}\right) \pm \epsilon_{i j} \partial_{j}\left(A_{i} W\right)\right] .
\end{aligned}
$$

Notably, $W\left(\phi_{n}\right)$ plays the role of a superpotential function, and it must be constructed (or proposed) such that the potential $U\left(\phi_{n}\right)$ becomes unity when $\phi_{n} \rightarrow 1$ (or $|\vec{x}| \rightarrow \infty$ ) in accordance with Eq. (16). Consequently, the following boundary conditions must be satisfied:

$$
\lim _{\phi_{n} \rightarrow 1} W\left(\phi_{n}\right)=0, \quad \lim _{\phi_{n} \rightarrow 1} \frac{\partial W}{\partial \phi_{n}}=0 .
$$

Then, under such boundary conditions we observe that the contributions of the total derivative in the second row of Eq. (21) vanish. Therefore, we can express the total energy as

$$
E=\bar{E}+E_{\mathrm{BPS}}
$$


where $\bar{E}$ represents the integral composed of the quadratic terms,

$\bar{E}=\int d^{2} \mathbf{x}\left[\frac{\left(B \pm \lambda^{2} g^{2} \mathcal{R} W\right)^{2}}{2 g^{2} \mathcal{R}}+\frac{\lambda^{2}}{2}\left(Q \mp \frac{\partial W}{\partial \phi_{n}}\right)^{2}\right]$,

and $E_{\mathrm{BPS}}$ defines the energy lower bound,

$$
E_{\mathrm{BPS}}= \pm \lambda^{2} \int d^{2} \mathbf{x}\left(\frac{\partial W}{\partial \phi_{n}}\right) \vec{\phi} \cdot\left(\partial_{1} \vec{\phi} \times \partial_{2} \vec{\phi}\right)
$$

The total energy (23) satisfies the inequality

$$
E \geq E_{\mathrm{BPS}}
$$

because $\bar{E} \geq 0$. Then, the energy lower bound will be achieved when the fields possess configurations such that $\bar{E}=0$, i.e., the bound is saturated when the following set of first-order differential equations is satisfied:

$$
\begin{gathered}
B=\mp \lambda^{2} g^{2} W\left(1-\frac{g^{2} \lambda^{4} W^{2}}{\beta^{2}}\right)^{-1 / 2}, \\
Q= \pm \frac{\partial W}{\partial \phi_{n}},
\end{gathered}
$$

where we have used

$$
\mathcal{R}=\left(1-\frac{g^{2} \lambda^{4} W^{2}}{\beta^{2}}\right)^{-1 / 2}
$$

This last equation, together with Eq. (20), allows us to write the self-dual potential of the model as

$\mathcal{V}\left(\phi_{n}\right)=\beta^{2}\left[1-\left(1-\frac{g^{2} \lambda^{4} W^{2}}{\beta^{2}}\right)^{1 / 2}\right]+\frac{\lambda^{2}}{2}\left(\frac{\partial W}{\partial \phi_{n}}\right)^{2}$.

Equations (27) and (28) are called the self-dual or BPS equations which ensure the energy lower bound and stability of the field configurations. Further, we highlight that such first-order equations satisfy the Euler-Lagrange equations associated with the Lagrangian density (2).

Before proceeding to the next section, we must highlight that the BPS configurations for the corresponding standard case (gauged BPS baby Skyrme model) can be recovered in the limit $\beta \rightarrow \infty$. In this limit, the BPS model described by the Lagrangian density (2) becomes

$$
\mathcal{L}=-\frac{1}{4 g^{2}} F_{\mu \nu}^{2}-\frac{\lambda^{2}}{4}\left(D_{\mu} \vec{\phi} \times D_{\nu} \vec{\phi}\right)^{2}-V\left(\phi_{n}\right),
$$

where the corresponding BPS potential is now given by

$$
V\left(\phi_{n}\right)=\frac{g^{2} \lambda^{4}}{2} W^{2}+\frac{\lambda^{2}}{2}\left(\frac{\partial W}{\partial \phi_{n}}\right)^{2}
$$

Such a system was investigated in Ref. [20].

\section{ROTATIONALLY SYMMETRIC BI SKYRMIONS}

We now consider rotationally symmetric solitons saturating the energy lower bound (25). Henceforth, without loss of generality, we set $\vec{n}=(0,0,1)$ such that $\phi_{n}=\phi_{3}$ and we assume the usual ansatz for the Skyrme field,

$$
\vec{\phi}(r, \theta)=\left(\begin{array}{c}
\sin f(r) \cos N \theta \\
\sin f(r) \sin N \theta \\
\cos f(r)
\end{array}\right),
$$

where $r$ and $\theta$ are polar coordinates, $N=\operatorname{deg}[\vec{\phi}]$ is the winding number introduced in Eq. (13), and $f(r)$ is a regular function satisfying the boundary conditions

$$
f(0)=\pi, \quad \lim _{r \rightarrow \infty} f(r)=0 .
$$

We now introduce the field redefinition [20]

$$
\phi_{3}=\cos f \equiv 1-2 h,
$$

with the field $h=h(r)$ obeying

$$
h(0)=1, \quad \lim _{r \rightarrow \infty} h(r)=0 .
$$

For the gauge field $A_{\mu}$, we consider the ansatz

$$
A_{i}=-\epsilon_{i j} x_{j} \frac{N a(r)}{r^{2}}
$$

where $a(r)$ is a regular function satisfying the boundary conditions

$$
a(0)=0, \quad \lim _{r \rightarrow \infty} a(r)=a_{\infty},
$$

where $a_{\infty}$ is a finite constant.

The superpotential $W(h)$ must satisfy

$\lim _{r \rightarrow 0} W(h)=W_{0}, \quad \lim _{r \rightarrow \infty} W(h)=0, \quad \lim _{r \rightarrow \infty} \frac{\partial W}{\partial h}=0$,

with the two last conditions obtained from Eq. (22). The constant $W_{0}=W(h(0))=W(1)$ is positive and finite. Besides, we consider the superpotential $W(h)$ a wellbehaved function for all of our analyses.

Under the ansatz, the BPS bound (25) becomes

$$
E \geq E_{\mathrm{BPS}}= \pm 2 \pi \lambda^{2} N W_{0},
$$


which is a positive-definite quantity, where the sign $+(-)$ corresponds to $N>0(N<0)$. The BPS energy density associated to this configuration may be written in the form

$$
\varepsilon_{\mathrm{BPS}}=g^{2} \lambda^{4} W^{2}\left(1-\frac{g^{2} \lambda^{4} W^{2}}{\beta^{2}}\right)^{-1 / 2}+\frac{\lambda^{2}}{4}\left(\frac{\partial W}{\partial h}\right)^{2} .
$$

Similarly, the BPS equations (27) and (28) assume, respectively, the forms

$$
\begin{gathered}
\frac{N}{r} \frac{d a}{d r}+\lambda^{2} g^{2} W\left(1-\frac{g^{2} \lambda^{4} W^{2}}{\beta^{2}}\right)^{-1 / 2}=0, \\
\frac{4 N}{r}(1+a) \frac{d h}{d r}+\frac{\partial W}{\partial h}=0,
\end{gathered}
$$

where we have used the magnetic field given by

$$
B=\frac{N}{r} \frac{d a}{d r} .
$$

Also note that, without loss of generality, we have chosen the upper sign. Such an assumption will be considered in the remainder of the manuscript.

In what follows, we present the behavior of the self-dual profiles close to the boundaries by solving the BPS equations (42) and (43) according to the already established boundary conditions. We begin by showing the behavior of the fields around the origin, which are given by

$$
\begin{gathered}
h(r) \approx 1-\frac{\left(W_{h}\right)_{h=1}}{8 N} r^{2}+\frac{\left(W_{h}\right)_{h=0}\left(W_{h h}\right)_{h=1}}{128 N^{2}} r^{4}, \\
a(r) \approx-\frac{\lambda^{2} g^{2} \mathcal{A}_{0} W_{0}}{2 N} r^{2}+\frac{\lambda^{2} g^{2} \mathcal{A}_{0}^{3}\left(W_{h}\right)_{h=1}^{2}}{32 N^{2}} r^{4},
\end{gathered}
$$

where $W_{h}=\partial W / \partial h, W_{h h}=\partial^{2} W / \partial h^{2}$, and the constant $\mathcal{A}_{0}$ is defined as

$$
\mathcal{A}_{0}=\left(1-\frac{g^{2} \lambda^{4} W_{0}^{2}}{\beta^{2}}\right)^{-1 / 2} .
$$

Furthermore, near the origin, for the magnetic field and BPS energy density we get, respectively,

$$
B(r) \approx-\lambda^{2} g^{2} \mathcal{A}_{0} W_{0}+\frac{\lambda^{2} g^{2} \mathcal{A}_{0}^{3}\left(W_{h}\right)_{h=1}^{2}}{8 N} r^{2},
$$

and

$\varepsilon_{\mathrm{BPS}} \approx \frac{\lambda^{2}\left(W_{h}\right)_{h=1}^{2}}{4}+\lambda^{4} g^{2} \mathcal{A}_{0} W_{0}^{2}-\frac{\lambda^{4} g^{2} \mathcal{A}_{1}\left(W_{h}\right)_{h=1}^{2}}{8 N} r^{2}$,

where $\mathcal{A}_{1}$ has been defined as

$$
\mathcal{A}_{1}=\frac{\left(W_{h h}\right)_{h=1}}{2 \lambda^{2} g^{2}}+\left(1+\mathcal{A}_{0}^{2}\right) \mathcal{A}_{0} W_{0} .
$$

The analysis of the behaviors near the origin of the gauge field profile (45), magnetic field (47), and BPS energy density (48) reveals the existence of a singularity associated with the BI parameter, as shown by the constant $\mathcal{A}_{0}$ defined in Eq. (46), i.e., the field profiles will be well defined if and only if the $\beta$ parameter satisfies

$$
\beta>\beta_{c}=g \lambda^{2} W_{0} .
$$

Thus, we expect that well-behaved solitons should exist in the range $\beta_{c}<\beta<\infty$, and that for sufficiently large values $\beta$, the soliton profiles become similar to those engendered by the model (31).

On the other hand, to compute the field profiles' behavior for sufficiently large values of $r$, we consider a superpotential $W(h)$ behaving as

$$
W(h) \approx W_{R}^{(\sigma)} h^{\sigma} \quad \text { with } \quad \sigma>1,
$$

where $W_{R}^{(\sigma)}>0$. The asymptotic form in Eq. (51) engenders the potential (30) behaving as $\mathcal{V} \sim h^{2 \sigma-2}$, allowing us to compare our solutions with those of the cases studied in Ref. [20].

Our analysis is performed by considering the boundary conditions

$$
h(R)=0, \quad a(R)=a_{R}, \quad W(h(R))=0,
$$

with $R>0$ and $a_{R}$ being a real constant. A finite value of $R$ defines the maximum size of the topological defect characterizing a soliton called compacton, i.e., it reaches the vacuum value in a finite radius $R$ (the compacton's radius) and remains in the vacuum for all $r>R$. On the other hand, when $R \rightarrow \infty$ the model engenders extended or noncompact configurations that may be localized or delocalized. Hence, we have different soliton configurations satisfying the boundary conditions (52). Of course, these solutions depend on the $\sigma$ values. In this way, the asymptotic analysis leads us to three types of solitons: (i) for $1<\sigma<2$ we have compactons; (ii) for $\sigma=2$ the soliton tail decays following a Gaussian law; (iii) for $\sigma>2$, the soliton tails have a power-law decay. Besides, these three types of soliton profiles behave near to the origin according as already presented in Eqs. (44) and (45).

We now calculate the magnetic flux of the BPS solitons. It is given by

$$
\Phi=2 \pi \int_{0}^{R} B r d r=2 \pi N a_{R}
$$

for compactons, whereas for the noncompact solitons the magnetic flux is 


$$
\Phi=2 \pi \int_{0}^{\infty} B r d r=2 \pi N a_{\infty} .
$$

We observe that the magnetic flux is nonquantized (in the topological sense) since $a_{R}$ or $a_{\infty}$ are real numbers belonging to the interval $\langle-1,0]$. However, for sufficiently large values of $g$, the vacuum value $a_{R}$ or $a_{\infty}$ tends to -1 ; consequently, in such a limit, the magnetic flux becomes quantized in units of $2 \pi$.

In the following sections, we show the field profiles' behavior in the limit in which the soliton reaches its corresponding vacuum value (i.e., $r \rightarrow R$ ) and also obtain the numerical solution of the BPS equations (42) and (43). Thus, we will choose specific superpotentials for such aims, allowing us to study the different types of solutions mentioned previously.

\section{A. Born-Infeld compactons}

In this section we approach a class of topological defects called compactons (i.e., solitons whose profiles reach the vacuum value at a finite distance). We point out that compact baby Skyrmions (nongauged case) were first investigated in Ref. [13]. Later, the gauged versions supporting purely magnetic compactons were studied in Ref. [20] and those carrying both magnetic flux and electric charge were studied in Ref. [24].

A superpotential like Eq. (51) for $r \rightarrow R$, i.e.,

$$
W(h) \approx W_{R} h^{\sigma} \quad \text { with } \quad 1<\sigma<2,
$$

with $R$ being a finite value, engenders Born-Infeld compactons whose profiles possess the following behavior:

$$
\begin{array}{r}
h(r) \approx \mathcal{C}_{R}(R-r)^{\frac{1}{2-\sigma}}+\frac{\mathcal{C}_{R}}{2(2-\sigma) R}(R-r)^{\frac{3-\sigma}{2-\sigma}}, \\
a(r) \approx a_{R}+\frac{2 g^{2} \lambda^{2}\left(1+a_{R}\right)\left(\mathcal{C}_{R}\right)^{2}}{\sigma}(R-r)^{\frac{2}{2-\sigma}}+\cdots \\
+\frac{g^{4} \lambda^{6} W_{R}^{2}\left(1+a_{R}\right)}{\beta^{2} \sigma(1+\sigma)}\left(\mathcal{C}_{R}\right)^{2+2 \sigma}(R-r)^{\frac{2+2 \sigma}{2-\sigma}},
\end{array}
$$

where we have considered the lowest order in $R-r$ and the first contribution of the BI parameter. We also have defined the quantity $\mathcal{C}_{R}$ as

$$
\mathcal{C}_{R}=\left[\frac{R \sigma(2-\sigma) W_{R}}{4 N\left(a_{R}+1\right)}\right]^{1 /(2-\sigma)},
$$

where $a_{R}=a(R)$ is the vacuum value of the gauge field profile.

The first relevant terms of the magnetic field and BPS energy density are given by

$$
\begin{aligned}
B= & -W_{R} g^{2} \lambda^{2}\left(\mathcal{C}_{R}\right)^{\sigma}(R-r)^{\frac{\sigma}{2-\sigma}}+\cdots \\
& -\frac{g^{4} \lambda^{6} W_{R}^{3}}{2 \beta^{2}}\left(\mathcal{C}_{R}\right)^{3 \sigma}(R-r)^{\frac{3 \sigma}{2-\sigma}}
\end{aligned}
$$

and

$$
\begin{aligned}
\varepsilon_{\mathrm{BPS}}= & \frac{W_{R}^{2} \lambda^{2}}{4} \sigma^{2}\left(\mathcal{C}_{R}\right)^{2 \sigma-2}(R-r)^{\frac{2 \sigma-2}{2-\sigma}}+\cdots \\
& +\frac{g^{4} \lambda^{8} W_{R}^{4}(2-\sigma)}{2 \beta^{2}(1+\sigma)}\left(\mathcal{C}_{R}\right)^{4 \sigma}(R-r)^{\frac{4 \sigma}{2-\sigma}}
\end{aligned}
$$

respectively.

For the numerical solutions, we select a superpotential like Eq. (55) and set $\sigma=3 / 2$. This way, we assume

$$
W(h)=W_{0} h^{3 / 2},
$$

by choosing $W_{0}=1 / \lambda^{2}$. This superpotential for $r \rightarrow R$ engenders a potential behaving as $\mathcal{V} \sim h$, which is analogous to the so-called "old baby Skyrme potential" [20]. For simplicity, we have fixed $N=1, \lambda=1$, and perform our numerical analysis in the following way: given a coupling constant $g$, we run distinct values for the BI parameter $\beta$. The compacton solutions are depicted in Figs. 1 and 2.

Once the parameter $\beta$ controls the BI term, which behaves as the Maxwell term for sufficiently large values of $\beta$, we expect the compacton profiles (colored lines) to be similar to those of the gauged BPS baby Skyrme model (black lines), as shown in Figs. 1 and 2. On the other hand, we observe that the compacton radius shrinks when $\beta$
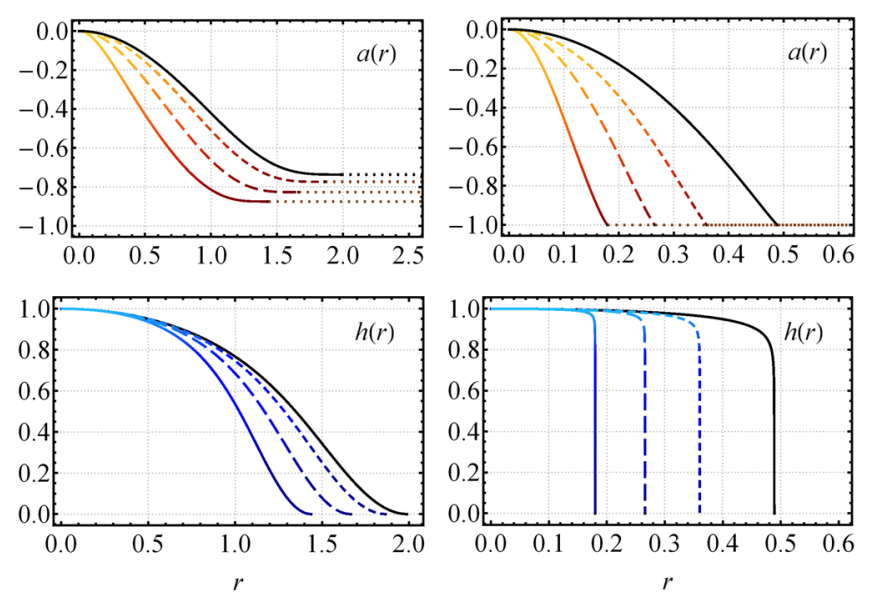

FIG. 1. The compacton profiles (color lines) generated by the superpotential (61) with $g=1$ (left), $g=3$ (right), and different values of $\beta$. Besides, we depict the corresponding ones fathered by the model (31) (solid black lines). For the Born-Infeld Skyrme model, we depict $\beta=\beta_{c}+0.01$ (solid lines), $\beta=\beta_{c}+0.1$ (long-dashed lines), and $\beta=\beta_{c}+0.5$ (dashed lines). The pointed lines in the profiles for the gauge field $a(r)$ stands for $r>R$ (outside of the compacton). 

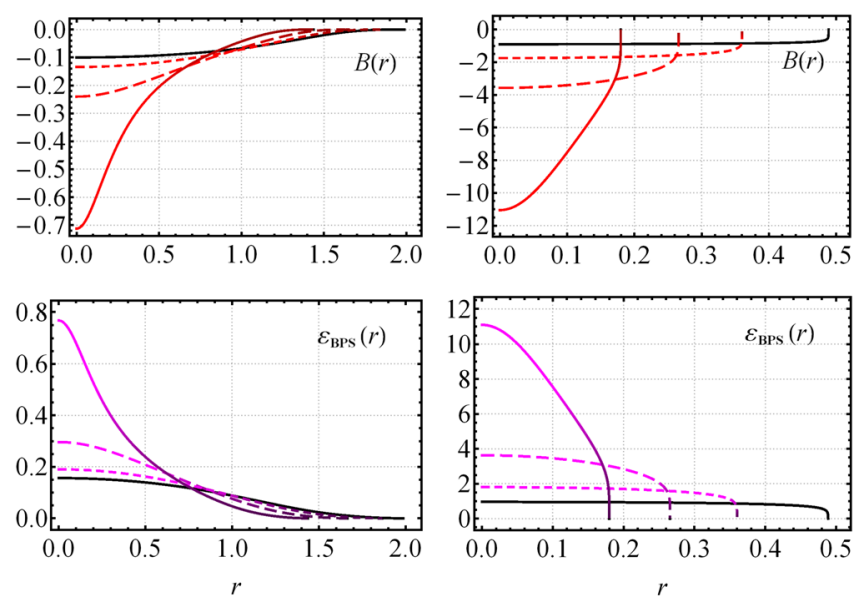

FIG. 2. Profiles for the magnetic field and BPS energy density rescaled by a factor of $10^{-1}$. Conventions are as in Fig. 1 .

decreases continuously but remains greater than the critical value $\beta_{c}$. Furthermore, we note that the compacton radius decreases when the value of $g$ increases (compare the left and right panels in Fig. 1), a general feature already observed in the solutions of its standard counterpart. Also, Fig. 2 shows that when the $\beta$ values are closer to $\beta_{c}$, the profiles of both to magnetic field and BPS energy density besides suffering relevant changes, the respective values overgrow at the origin.

We point out that, for our analysis, we have conveniently considered the values $(g \geq 1)$. This is because when $g$ is small, the nonlinear effects of the BI term become insignificant, which is clarified by looking at Eq. (42) where the term in brackets depending on $\beta$ becomes higher order than $g^{2}$ due to the constraint (50). In this way, for sufficiently small values of $g$, the soliton profiles become similar to those of the standard case described by the Lagrangian density given in Eq. (31). Similar considerations also arise for the case of the extended solitons, which we will analyze in the following sections.

\section{B. Localized Born-Infeld Skyrmions}

As already commented, the model provides noncompact configurations when the vacuum value happens at $R \rightarrow \infty$. This way, for superpotentials like Eq. (51) with $\sigma=2$, i.e.,

$$
W(h) \approx W_{\infty}^{(2)} h^{2},
$$

the resulting extended configurations represent localized solitons, whose field profiles behave as

$$
h(r) \approx \mathcal{C}_{\infty}^{(2)} e^{-\Lambda r^{2}}
$$

$$
\begin{aligned}
a(r) \approx & a_{\infty}+\lambda^{2} g^{2}\left(1+a_{\infty}\right)\left(\mathcal{C}_{\infty}^{(2)}\right)^{2} e^{-2 \Lambda r^{2}} \\
& +\frac{g^{4} \lambda^{6}\left(1+a_{\infty}\right)\left(W_{\infty}^{(2)}\right)^{2}}{6 \beta^{2}}\left(\mathcal{C}_{\infty}^{(2)}\right)^{6} e^{-6 \Lambda r^{2}},
\end{aligned}
$$

where we have considered the first contribution of the BI parameter. Besides $\mathcal{C}_{\infty}^{(2)}>0$, the parameter $a_{\infty}=a(\infty)$ is the vacuum value of the gauge field profile, and $\Lambda>0$ is given by

$$
\Lambda=\frac{W_{\infty}^{(2)}}{4 N\left(1+a_{\infty}\right)} .
$$

Further, we can exhibit the corresponding behaviors for both the magnetic field and BPS energy density, which read as

$$
\begin{aligned}
B(r) \approx & -\lambda^{2} g^{2} W_{\infty}^{(2)}\left(\mathcal{C}_{\infty}^{(2)}\right)^{2} e^{-2 \Lambda r^{2}}+\cdots \\
& -\frac{g^{4} \lambda^{6}\left(W_{\infty}^{(2)}\right)^{3}\left(\mathcal{C}_{\infty}^{(2)}\right)^{6}}{2 \beta^{2}} e^{-6 \Lambda r^{2}}
\end{aligned}
$$

and

$$
\begin{aligned}
\varepsilon_{\mathrm{BPS}} \approx & \lambda^{2}\left(W_{\infty}^{(2)}\right)^{2}\left(\mathcal{C}_{\infty}^{(2)}\right)^{2} e^{-2 \Lambda r^{2}}+\cdots \\
& +\frac{g^{4} \lambda^{8}\left(W_{\infty}^{(2)}\right)^{4}\left(\mathcal{C}_{\infty}^{(2)}\right)^{8}}{2 \beta^{2}} e^{-8 \Lambda r^{2}},
\end{aligned}
$$

respectively.

For our numerical analysis, we set the superpotential to

$$
W(h)=W_{0} h^{2},
$$

by assuming $W_{0}=1 / \lambda^{2}$. The superpotential for $r \rightarrow \infty$ provides a potential behaving as $\mathcal{V} \sim h^{2}$, which also has an analogous version investigated in Ref. [20]. As in the previous case, we adopt $N=1$ and $\lambda=1$, and we run distinct values for the BI parameter $\beta$ for a fixed value of the coupling constant $g$. The numerical solutions depicted in Figs. 3 and 4 show the field profiles, the magnetic field, and BPS energy density. Observing the profiles, we perceive that they present many previously discussed features for the compacton's case. However, here the solitons are extended and localized, and for sufficiently large values of $r$ their tails decay following a Gaussian law.

\section{Delocalized Born-Infeld Skyrmions}

We now consider a superpotential whose behavior for $r \rightarrow \infty$ is given by Eq. (51) with $\sigma>2$,

$$
W(h) \approx W_{\infty}^{(\sigma)} h^{\sigma},
$$



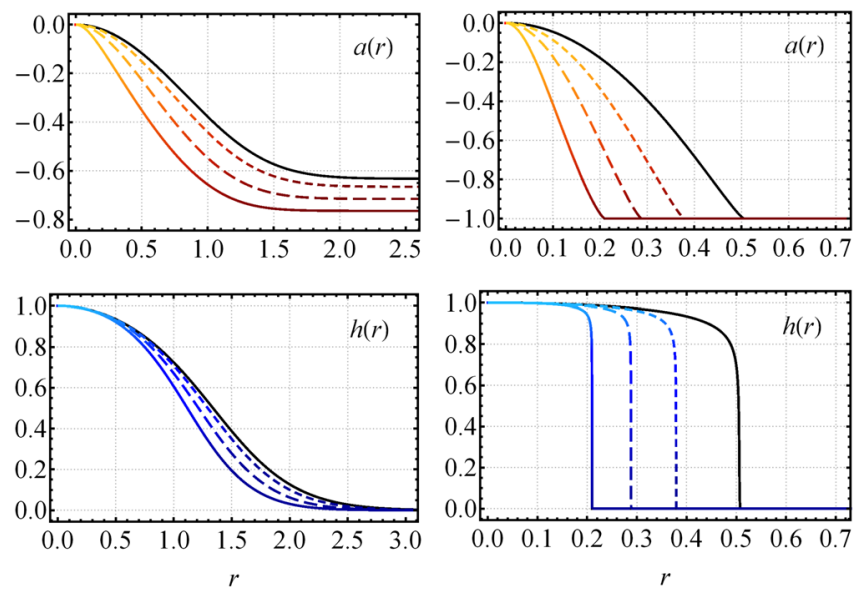

FIG. 3. Profiles for the solitons by assuming the superpotential (68). Conventions are as in Fig. 1.

where $W_{\infty}^{(\sigma)}$ is a positive constant. Then, in this limit, the field profiles have the asymptotic behavior

$$
\begin{gathered}
h(r) \approx\left(\frac{\mathcal{C}_{\infty}^{(\sigma)}}{r^{2}}\right)^{\frac{1}{\sigma-2}} \\
a(r) \approx a_{\infty}+\frac{2 g^{2} \lambda^{2}\left(1+a_{\infty}\right)}{\sigma}\left(\frac{\mathcal{C}_{\infty}^{(\sigma)}}{r^{2}}\right)^{\frac{2}{\sigma-2}}+\cdots \\
+\frac{g^{4} \lambda^{6}\left(1+a_{\infty}\right)\left(W_{\infty}^{(\sigma)}\right)^{2}}{\sigma(\sigma+1) \beta^{2}}\left(\frac{\mathcal{C}_{\infty}^{(\sigma)}}{r^{2}}\right)^{\frac{2 \sigma+2}{\sigma-2}}
\end{gathered}
$$

where we have considered the lowest order in $r^{-1}$ and the first contribution of the BI parameter. The constant $\mathcal{C}_{\infty}^{(\sigma)}$ is given by

$$
\mathcal{C}_{\infty}^{(\sigma)}=\frac{8 N\left(a_{\infty}+1\right)}{\sigma(\sigma-2) W_{\infty}^{(\sigma)}}
$$
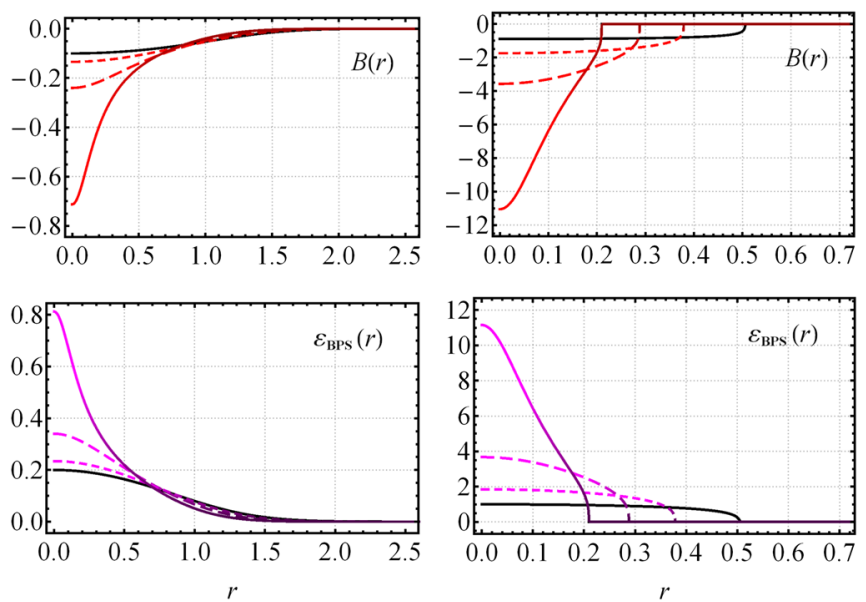

FIG. 4. Profiles for the solitons by assuming the superpotential (68). Conventions are as in Fig. 2. remembering that the parameter $a_{\infty}=a(\infty)$ is the vacuum value of the gauge field profile. Furthermore, the magnetic field and BPS energy density behave as

$$
\begin{aligned}
B(r) \approx & -\lambda^{2} g^{2} W_{\infty}^{(\sigma)}\left(\frac{\mathcal{C}_{\infty}^{(\sigma)}}{r^{2}}\right)^{\frac{\sigma}{\sigma-2}}+\cdots \\
& -\frac{g^{4} \lambda^{6}\left(W_{\infty}^{(\sigma)}\right)^{3}}{2 \beta^{2}}\left(\frac{C_{\infty}^{(\sigma)}}{r^{2}}\right)^{\frac{3 \sigma}{\sigma-2}}
\end{aligned}
$$

and

$$
\begin{aligned}
\varepsilon_{\mathrm{BPS}} \approx & \frac{1}{4} \sigma^{2} \lambda^{2}\left(W_{\infty}^{(\sigma)}\right)^{2}\left(\frac{\mathcal{C}_{\infty}^{(\sigma)}}{r^{2}}\right)^{\frac{2 \sigma-2}{\sigma-2}}+\cdots \\
& +\frac{g^{4} \lambda^{8}\left(W_{\infty}^{(2)}\right)^{4}}{2 \beta^{2}}\left(\frac{\mathcal{C}_{\infty}^{(\sigma)}}{r^{2}}\right)^{\frac{4 \sigma}{\sigma-2}},
\end{aligned}
$$

respectively.

To perform the numerical analysis, we select the superpotential

$$
W(h)=W_{0} h^{\sigma}, \quad \sigma>2
$$

by setting $W_{0}=1 / \lambda^{2}$. Next, by considering $N=1, \lambda=1$, and fixing both of the coupling constants $g$ and $\beta$, we run distinct values for the parameter $\sigma$. We adopt a different approach than in the two previous cases because we want to analyze the soliton's features for $\sigma>2$. With such a perspective, the numerical solutions depicted in Fig. 5 show the field profiles, the magnetic field, and the BPS energy density.

We observe that the behavior of the Skyrme field profiles $h(r)$ follow a decay more slowly to its vacuum value whenever $\sigma$ increases by following the power law presented in Eq. (70), see the upper left panel in Fig. 5. We also see that the Skyrme field profiles and the corresponding ones for the
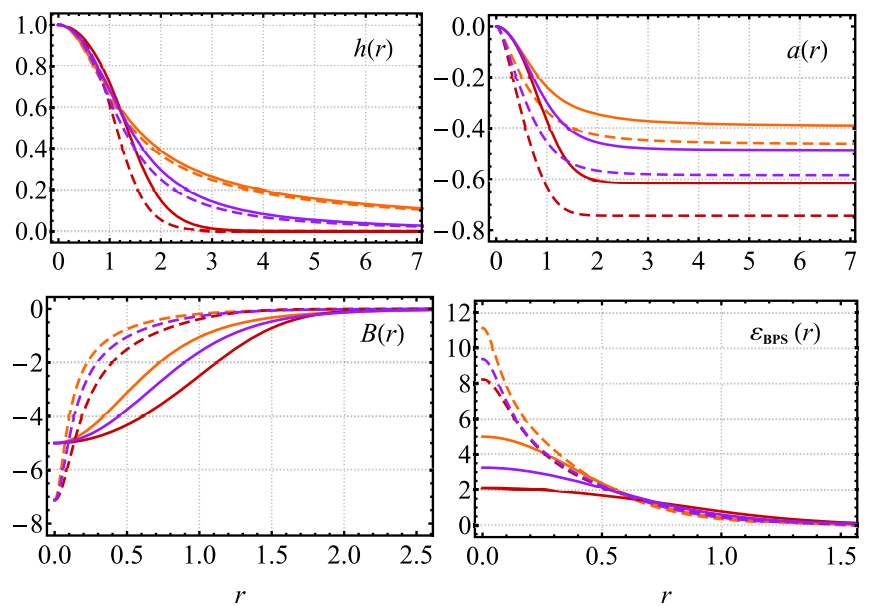

FIG. 5. Profiles for the solitons (dashed lines) generated by the superpotential (75) with $g=1, \beta=1.01$, and distinct values of $\sigma$ which are exhibited together with those of the counterpart model (31) (solid lines). We show the profiles with $\sigma=2.1$ (red lines), $\sigma=3.0$ (purple lines), and $\sigma=4.0$ (orange lines). The $B(r)$ profiles for the standard case have been rescaled by a factor of 5 . 
gauged BPS baby Skyrme model, Eq. (31), become closer and closer as $\sigma$ grows. The same effect is also observed in the gauge field profiles $a(r)$, see the upper right panel in Fig. 5. On the other hand, the magnetic field and BPS energy density exhibit distinct values at the origin and different formats to those of the model defined by Eq. (31). Interestingly, for every model, unlike the BPS energy density, the magnetic field has the same values at the origin for all values of $\sigma$. This happens because of our choice $\lambda=1$ (hence, $W_{0}=1$ ) in all cases, verifying the result shown in Eq. (47).

\section{CONCLUSIONS AND REMARKS}

We have shown that topological BPS Skyrmions exist in a restricted baby Skyrme model endowed with a generalized gauge field dynamic given by the BornInfeld term. The term possesses a free parameter $\beta$, which for sufficiently large values reproduces effects similar to the Maxwell term. Next, the Bogomol'nyi framework's successful implementation provides both an energy lower bound and the corresponding self-dual or BPS equations, whose solutions are the fields saturating such a lower limit. The behavior of the field profiles near the origin reveals the existence of a critical value [Eq. (50)] of the BI parameter, i.e., only above such a value can we obtain well-behaved solitons. On the other hand, depending on the behavior of the superpotential at large values of $r$, the model supports three different types of soliton solutions. Finally, by choosing specific superpotentials, we have obtained numerical solutions of the BPS equations, and we compared the resulting solitons with those of the standard counterpart.

In Figs. 1 and 2 we have shown the Born-Infeld compactons for different values of $\beta$, given a fixed value of $g$. The figures allow us to conclude that the soliton becomes more compacted whenever the value of $\beta$ decreases. The left panel in Fig. 6 depicts this property by clearly showing the dependence of the compacton radius $R$ on $\beta$. We note that the radius becomes smaller and smaller as $\beta$ gets closer to the critical value $\beta_{c}=1$ and, since $\beta>\beta_{c}$, there is a minimum radius $R_{\min }^{(c)}>0$ such that $R \rightarrow R_{\min }^{(c)}$ asymptotically when $\beta \rightarrow \beta_{c}$. On the other hand, the soliton grows larger when $\beta$
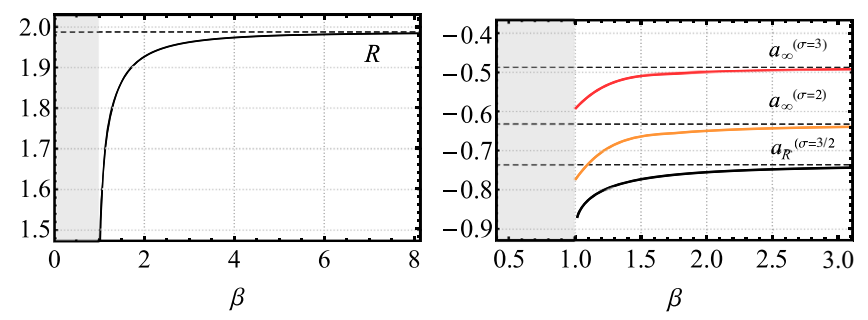

FIG. 6. Left: compacton radius $R$ vs $\beta$. Right: gauge field vacuum value vs $\beta$ for the three distinct superpotentials analyzed here. We set $N=1, g=1$, and $\lambda=1$. The dashed lines depict the standard counterparts.
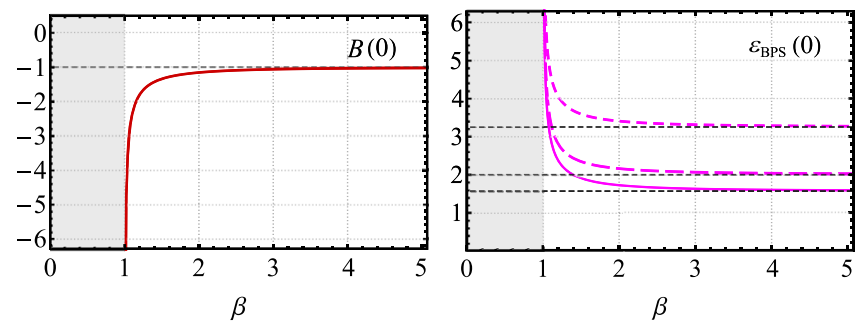

FIG. 7. Magnetic field (left) and BPS energy density (right) amplitudes at the origin as functions of the BI parameter $\beta$, with $N=1, g=1$, and $\lambda=1$. The pictures depict both behaviors for the solitons coming from the superpotentials with $\sigma=3 / 2$ (solid line), $\sigma=2$ (long-dashed line), and $\sigma=3$ (dashed line), with the respective standard counterpart (black dashed lines).

increases continuously until the maximum size of the soliton approaches that of the standard case.

Besides the compacton solitons, the model has two other types of solutions that we named extended or noncompact solitons, which also present some general features already described for the compactons, e.g., $\beta$ controls how the solutions move away from or approach the respective standard counterparts. The right panel of Fig. 6 shows the evolution of the vacuum value of the gauge field profiles of the three types of Born-Infeld Skyrmions as $\beta$ grows. It is clear that the values shift toward those of the respective gauged BPS baby Skyrme model (dashed black lines). In addition, this plot also shows how the magnetic flux (53) in units of $2 \pi N$ changes as a function of $\beta$.

The values of the magnetic field at the origin are independent of the $\sigma$ parameter according to Eq. (47). This is shown in the bottom-left panel of Fig. 5 for the solutions with $\sigma>2$, and the left panel of Fig. 7 shows the $B(0)$ amplitudes for all of the superpotentials that coincide on a single curve. On the other hand, the BPS energy density $\varepsilon_{\mathrm{BPS}}(0)$ possesses an explicit dependence on $\sigma$ as given by the first term of Eq. (48); see the lower-right panel of Fig. 5 and the right panel of Fig. 7.

Finally, we are currently studying the possible existence of charged BPS Skyrmions (maybe behaving as anions) in the presence of dielectric media or magnetic impurities. Advances in this direction will be reported elsewhere.

\section{ACKNOWLEDGMENTS}

This study was financed in part by the Coordenação de Aperfeiçoamento de Pessoal de Nível Superior-Brasil (CAPES)_Finance Code 001. We also thank the Conselho Nacional de Desenvolvimento Científico e Tecnológico $(\mathrm{CNPq})$, and the Fundação de Amparo à Pesquisa e ao Desenvolvimento Científico e Tecnológico do Maranhão (FAPEMA) (Brazilian Government agencies). In particular, A. C. S. thanks the full support from CAPES. R. C. acknowledges the support from the grants CNPq/ 306724/2019-7, CNPq/423862/2018-9, FAPEMA/ Universal-01131/17, and FAPEMA/Universal-00812/19. 
[1] T. H. R. Skyrme, Proc. R. Soc. A 260, 127 (1961); Nucl. Phys. 31, 556 (1962); J. Math. Phys. (N. Y.) 12, 1735 (1971).

[2] G. S. Adkins, C. R. Nappi, and E. Witten, Nucl. Phys. B228, 552 (1983); G. S. Adkins and C. R. Nappi, Nucl. Phys. B233, 109 (1984); C. J. Halcrow, C. King, and N. S. Manton, Phys. Rev. C 95, 031303(R) (2017); C. Naya and P. Sutcliffe, Phys. Rev. Lett. 121, 232002 (2018); I. Sharma, R. Kumar, and M. K. Sharma, Nucl. Phys. A983, 276 (2019).

[3] B. M. A. G. Piette, B. J. Schroers, and W. J. Zakrzewski, Z. Phys. C 65, 165 (1995); Nucl. Phys. B439, 205 (1995).

[4] S. L. Sondhi, A. Karlhede, S. A. Kivelson, and E. H. Rezayi, Phys. Rev. B 47, 16419 (1993); O. Schwindt and N. R. Walet, Europhys. Lett. 55, 633 (2001); A. Neubauer, C. Pfleiderer, B. Binz, A. Rosch, R. Ritz, P. G. Niklowitz, and P. Böni, Phys. Rev. Lett. 102, 186602 (2009); A.C. Balram, U. Wurstbauer, A. Wojs, A. Pinczuk, and J. K. Jain, Nat. Commun. 6, 8981 (2015); T. Chen and T. Byrnes, Phys. Rev. B 99, 184427 (2019).

[5] J. Fukuda and S. Zumer, Nat. Commun. 2, 246 (2011); S. Kang, E.-W. Lee, T. Li, X. Liang, M. Tokita, K. Nakajima, and J. Watanabe, Angew. Chem. Int. Ed. 55, 11552 (2016).

[6] A. A. Zyuzin, J. Garaud, and E. Babaev, Phys. Rev. Lett. 119, 167001 (2017).

[7] Y. Kodama, K. Kokubu, and N. Sawado, Phys. Rev. D 79, 065024 (2009); Y. Brihaye, T. Delsate, Y. Kodama, and N. Sawado, Phys. Rev. D 82, 106002 (2010); T. Delsate and N. Sawado, Phys. Rev. D 85, 065025 (2012).

[8] S. Mühlbauer, B. Binz, F. Jonietz, C. Pfleiderer, A. Rosch, A. Neubauer, R. Georgii, and P. Boni, Science 323, 915 (2009); X. Z. Yu, Y. Onose, N. Kanazawa, J. H. Park, J. H. Han, Y. Matsui, N. Nagaosa, and Y. Tokura, Nature (London) 465, 901 (2010).

[9] T. Dohi, S. DuttaGupta, S. Fukami, and H. Ohno, Nat. Commun. 10, 5153 (2019).

[10] B. J. Schroers, SciPost Phys. 7, 030 (2019); B. BartonSinger, C. Ross, and B. J. Schroers, Commun. Math. Phys. 375, 2259 (2020).

[11] R. H. Hobart, Proc. Phys. Soc. 82, 201 (1963).

[12] G. H. Derrick, J. Math. Phys. (N.Y.) 5, 1252 (1964).

[13] T. Gisiger and M. B. Paranjape, Phys. Rev. D 55, 7731 (1997).

[14] C. Adam, T. Romanczukiewicz, J. Sanchez-Guillen, and A. Wereszczynski, Phys. Rev. D 81, 085007 (2010).

[15] E. Witten, Nucl. Phys. B223, 422 (1983); B223, 433 (1983); C. G. Callan and E. Witten, Nucl. Phys. B239, 161 (1984).

[16] J. Gladikowski, B. M. A. G. Piette, and B. J. Schroers, Phys. Rev. D 53, 844 (1996).

[17] A. Samoilenka and Ya. Shnir, Phys. Rev. D 93, 065018 (2016).

[18] A. Samoilenka and Ya. Shnir, Phys. Rev. D 95, 045002 (2017).

[19] F. Navarro-Lerida, E. Radu, and D. H. Tchrakian, Phys. Rev. D 95, 085016 (2017).

[20] C. Adam, C. Naya, J. Sanchez-Guillen, and A. Wereszczynski, Phys. Rev. D 86, 045010 (2012).

[21] C. Adam, C. Naya, T. Romanczukiewicz, J. SanchezGuillen, and A. Wereszczynski, J. High Energy Phys. 05 (2015) 155.
[22] C. Adam and A. Wereszczynski, Phys. Rev. D 95, 116006 (2017).

[23] C. Adam, C. Naya, J. Sanchez-Guillen, and A. Wereszczynski, J. High Energy Phys. 03 (2013) 012.

[24] R. Casana, A. C. Santos, C. F. Farias, and A. L. Mota, Phys. Rev. D 100, 045022 (2019).

[25] R. Casana, A. C. Santos, C. F. Farias, and A. L. Mota, Phys. Rev. D 101, 045018 (2020).

[26] S. Bolognesi and S. B. Gudnason, Nucl. Phys. B805, 104 (2008).

[27] S. Bolognesi and W. Zakrzewski, Phys. Rev. D 91, 045034 (2015).

[28] C. Adam, J. M. Queiruga, J. Sanchez-Guillen, and A. Wereszczynskic, J. High Energy Phys. 05 (2013) 108; J. M. Queiruga, Phys. Rev. D 92, 105012 (2015).

[29] M. Nitta and S. Sasaki, Phys. Rev. D 90, 105002 (2014); 91, 125025 (2015); S. B. Gudnason, M. Nitta, and S. Sasaki, J. High Energy Phys. 01 (2017) 014.

[30] J. M. Queiruga, J. Phys. A 52, 055202 (2019).

[31] E. Babichev, Phys. Rev. D 74, 085004 (2006); 77, 065021 (2008).

[32] D. Bazeia, L. Losano, R. Menezes, and J. C. R. E. Oliveira, Eur. Phys. J. C 51, 953 (2007).

[33] E. Babichev, P. Brax, C. Caprini, J. Martin, and D. A. Steer, J. High Energy Phys. 03 (2009) 091.

[34] D. Bazeia, E. da Hora, and D. Rubiera-Garcia, Phys. Rev. D 84, 125005 (2011).

[35] R. Casana, E. da Hora, D. Rubiera-Garcia, and C. dos Santos, Eur. Phys. J. C 75, 380 (2015).

[36] R. Casana, A. Cavalcante, and E. da Hora, J. High Energy Phys. 12 (2016) 051.

[37] M. Kuniyasu, N. Sakai, and K. Shiraishi, Phys. Rev. D 94, 116001 (2016).

[38] C. Adam, N. Grandi, P. Klimas, J. Sanchez-Guillen, and A. Wereszczynski, J. Phys. A 41, 375401 (2008).

[39] C. Adam, J. M. Queiruga, J. Sanchez-Guillen, and A. Wereszczynski, Phys. Rev. D 84, 065032 (2011).

[40] C. Adam, J. M. Queiruga, J. Sanchez-Guillen, and A. Wereszczynski, Phys. Rev. D 84, 025008 (2011).

[41] M. Koehn and M. Trodden, Phys. Lett. B 755, 498 (2016).

[42] C. Armendariz-Picon, T. Damour, and V. Mukhanov, Phys. Lett. B 458, 209 (1999).

[43] V. Mukhanov and A. Vikman, J. Cosmol. Astropart. Phys. 02 (2006) 004.

[44] A. Sen, J. High Energy Phys. 07 (2002) 065.

[45] C. Armendariz-Picon and E. A. Lim, J. Cosmol. Astropart. Phys. 08 (2005) 007.

[46] K. A. Bronnikov and J. C. Fabris, Phys. Rev. Lett. 96, 251101 (2006).

[47] V. Faraoni and A. Jacques, Phys. Rev. D 76, 063510 (2007).

[48] J. Garriga and V. F. Mukhanov, Phys. Lett. B 458, 219 (1999).

[49] R. J. Scherrer, Phys. Rev. Lett. 93, 011301 (2004).

[50] M. Born and L. Infeld, Proc. R. Soc. A 144, 425 (1934).

[51] P. A. M. Dirac, Proc. R. Soc. A 268, 57 (1962).

[52] K. Shiraishi and S. Hirenzaki, Int. J. Mod. Phys. A 06, 2635 (1991).

[53] E. Moreno, C. Nunez, and F. A. Schaposnik, Phys. Rev. D 58, 025015 (1998). 
[54] B. Dion, L. Marleau, and G. Simon, Phys. Rev. D 53, 1542 (1996).

[55] O. V. Pavlovskii, Phys. Lett. B 538, 202 (2002).

[56] G. Boillat, J. Math. Phys. (N.Y.) 11, 941 (1970).

[57] A. Garcia, H. Salazar, and J. F. Plebanski, Nuovo Cimento 84, 65 (1984).

[58] T. K. Dey, Phys. Lett. B 595, 484 (2004).
[59] J. Ellis, N. E. Mavromatos, and T. You, Phys. Rev. Lett. 118, 261802 (2017).

[60] E. Fradkin and A. A. Tseytlin, Phys. Lett. 163B, 123 (1985).

[61] D. Brecher, Phys. Lett. B 442, 117 (1998).

[62] A. A. Tseytlin, Nucl. Phys. B501, 41 (1997).

[63] S. Sarangi, J. High Energy Phys. 07 (2008) 018. 